\title{
Copy number alterations in cancer: detection and precision medicine
}

\author{
Arief Gusnanto ${ }^{1}$ \\ ${ }^{1}$ Department of Statistics, University of Leeds, UK
}

\begin{abstract}
Copy number alterations (CNAs) are genomic alterations where some regions exhibit more or less copy number than the normal two copies. In this talk, I will describe two ideas: (1) how CNAs are estimated from data generated by next generation sequencing (NGS) and what steps are required to make the data interpretable, (2) how the CNA can be utilised for precision medicine in terms of prediction of tumour subtypes and prediction of cancer patients' survival. If time permits, I will also discuss how to estimate genomic markers from CNA profile across cancer patients.
\end{abstract}

